\title{
Existence and Uniqueness of Pseudo Almost Automorphic Mild Solutions to Some Classes of Partial Hyperbolic Evolution Equations
}

\author{
Zhanrong $\mathrm{Hu}^{1,2}$ and Zhen Jin ${ }^{1,2}$ \\ ${ }^{1}$ Department of Mathematics, North University of China, Taiyuan 030051, China \\ ${ }^{2}$ School of Mechatronic Engineering, North University of China, Taiyuan 030051, China \\ Correspondence should be addressed to Zhanrong Hu, huzhanrong22@126.com \\ Received 1 June 2008; Revised 4 July 2008; Accepted 10 September 2008 \\ Recommended by Leonid Shaikhet
}

We will establish an existence and uniqueness theorem of pseudo almost automorphic mild solutions to the following partial hyperbolic evolution equation $(d / d t)[u(t)+f(t, B u(t))]=$ $A u(t)+g(t, C u(t)), t \in \mathbb{R}$, under some assumptions. To illustrate our abstract result, a concrete example is given.

Copyright (C) $2008 \mathrm{Z}$. Hu and Z. Jin. This is an open access article distributed under the Creative Commons Attribution License, which permits unrestricted use, distribution, and reproduction in any medium, provided the original work is properly cited.

\section{Introduction}

Let $(\mathbb{X},\|\cdot\|)$ be a Banach space, and let $\left(\mathbb{X}_{\alpha},\|\cdot\|_{\alpha}\right)$ for $0<\alpha<1$ be an arbitrary abstract intermediate Banach space between $D(A)$ (domain of the operator $A$ ) and $\mathbb{X}$. In this paper, we deal with sufficient conditions for the existence and uniqueness of pseudo almost automorphic mild solutions to equation

$$
\frac{d}{d t}[u(t)+f(t, B u(t))]=A u(t)+g(t, C u(t)), \quad t \in \mathbb{R},
$$

where $A$ is a sectorial linear operator on a Banach space $\mathbb{X}$ and $\sigma(A) \cap i \mathbb{R}=\varnothing, B, C$ are bounded linear operators on $\mathbb{X}_{\alpha}, f: \mathbb{R} \times \mathbb{X} \mapsto \mathbb{X}_{\beta}, g: \mathbb{R} \times \mathbb{X} \mapsto \mathbb{X}$ are jointly continuous functions. This turns out to be a nontrivial problem due to the complexity and importance of pseudo almost automorphic functions (e.g., see [1-8] and the references therein).

Upon making some additional assumptions, it will be shown that (1.1) admits a unique $\mathbb{X}_{\alpha}$-valued pseudo almost automorphic mild solution. Applications include the study 
of pseudo almost automorphic mild solutions to some nonlinear heat equation:

$$
\begin{gathered}
u_{t}(t, x)=u_{x x}(t, x)+a u(t, x)+g(t, q(x) u(t, x)), \quad t \in \mathbb{R}, x \in[0, \pi], \\
u(t, 0)=u(t, \pi)=0, \quad t \in \mathbb{R},
\end{gathered}
$$

where $a \in \mathbb{R}$ is a constant, and $g, q$ are continuous functions.

As a natural and important generalization of almost automorphy as well as pseudo almost periodicity, pseudo almost automorphy has recently been investigated (for more details, see [1-3]). Moreover, Liang et al. in [2] established a composition theorem about pseudo almost automorphic functions; Xiao et al. in [3] obtained sufficient conditions for the existence and uniqueness of pseudo almost automorphic mild solutions to semilinear differential equations in Banach spaces. In particular, they proved in [3] that the space $\left(\operatorname{PAA}(\mathbb{R}, \mathbb{X}),\|\cdot\|_{\infty}\right)$ of pseudo almost automorphic functions, endowed with sup norm, is a Banach space, thus pushed the door open to study pseudo almost automorphic mild solutions to various differential equations.

The method used here was firstly developed in [9], in which Diagana established an existence and uniqueness theorem of pseudo almost periodic mild solutions to (1.1) under some similar assumptions. Combining bounded invariance theorem of pseudo almost automorphic functions established in [6] with composition theorem of pseudo almost automorphic functions and completeness of the space $\left(\operatorname{PAA}(\mathbb{R}, \mathbb{X}),\|\cdot\|_{\infty}\right)$, this paper extends the results in [9], since such function as $g(t, u)=u \sin (1 /(2+\cos t+\cos \sqrt{2} t))+$ $\max _{k \in \mathbb{Z}}\left\{e^{-\left(t \pm k^{2}\right)^{2}}\right\} \cos u, t \in \mathbb{R}, u \in \mathbb{X}$ is not pseudo almost periodic, while they are pseudo almost automorphic.

It should be pointed out that one can also make use of (1.1) to study several types of evolution equations including partial functional differential equations, integro-differential equations, and reaction diffusion equations.

\section{Preliminaries}

In this section, we collect some preliminary facts from [9-11] that will be used later. Throughout this paper, $\mathbb{N}, \mathbb{Z}, \mathbb{R}$, and $\mathbb{C}$ stand for the sets of positive integer, integer, real and complex numbers; $(\mathbb{X},\|\cdot\|),\left(\mathbb{Y},\|\cdot\|_{\mathbb{Y}}\right)$ stand for Banach spaces. If $A$ is a linear operator on $\mathbb{X}$, then $\rho(A), \sigma(A), D(A), \operatorname{ker}(A), R(A)$ stand for the resolvent set, spectrum, domain, kernel, and range of $A$. The space $B(\mathbb{X}, \mathbb{Y})$ denotes the Banach space of all bounded linear operators from $\mathbb{X}$ into $\mathbb{Y}$ equipped with natural norm $\|A\|_{B(\mathbb{X}, \mathbb{Y})}=\sup _{x \in \mathbb{X}, x \neq 0}\left(\|A x\|_{\mathbb{Y}} /\|x\|\right)$. If $\mathbb{Y}=\mathbb{X}$, it is simply denoted by $B(\mathbb{X})$ with $\|A\|_{B(\mathbb{X})}=\sup _{x \in \mathbb{X}, x \neq 0}(\|A x\| /\|x\|)$.

\subsection{Sectorial linear operators and analytic semigroups}

Definition 2.1. A linear operator $A: D(A) \subset \mathbb{X} \mapsto \mathbb{X}$ (not necessarily densely defined) is said to be sectorial if the following hold. There exist constants $\omega \in \mathbb{R}, \theta \in(\pi / 2, \pi)$, and $M>0$ such that

$$
\begin{gathered}
\rho(A) \supset S_{\theta, \omega}:=\{\lambda \in \mathbb{C}: \lambda \neq \omega,|\arg (\lambda-\omega)|<\theta\}, \\
\|R(\lambda, A)\| \leq \frac{M}{|\lambda-\omega|}, \quad \lambda \in S_{\theta, \omega}
\end{gathered}
$$

where $R(\lambda, A)=(\lambda I-A)^{-1}$ for each $\lambda \in \rho(A)$. 
Remark 2.2. If $A$ is sectorial, then it generates an analytic semigroup $(T(t))_{t \geq 0}$, which maps $(0, \infty)$ into $B(\mathbb{X})$ such that there exist $M_{0}, M_{1}>0$ with

$$
\begin{gathered}
\|T(t)\| \leq M_{0} e^{\omega t}, \quad t>0, \\
\|t(A-\omega I) T(t)\| \leq M_{1} e^{\omega t}, \quad t>0 .
\end{gathered}
$$

Definition 2.3. A semigroup $(T(t))_{t \geq 0}$ is said to be hyperbolic, if there exist a projection $P$ and constants $M, \delta>0$ such that each $T(t)$ commutes with $P, \operatorname{ker}(P)$ is invariant with respect to $T(t), T(t): R(Q) \rightarrow R(Q)$ is invertible and

$$
\begin{array}{cc}
\|T(t) P x\| \leq M e^{-\delta t}\|x\| & \text { for } t \geq 0, \\
\|T(t) Q x\| \leq M e^{\delta t}\|x\| & \text { for } t \leq 0,
\end{array}
$$

where $Q:=I-P$ and $T(t):=(T(-t))^{-1}$ for $t \leq 0$.

Recall that if a semigroup $(T(t))_{t \geq 0}$ is analytic, then $(T(t))_{t \geq 0}$ is hyperbolic if and only if $\sigma(A) \cap i \mathbb{R}=\varnothing$ (see, e.g., [11, Definition 1.14 and Proposition 1.15, page 305]).

\subsection{Intermediate Banach spaces}

Definition 2.4. Let $\alpha \in(0,1)$. A Banach space $\left(\mathbb{X}_{\alpha},\|\cdot\|_{\alpha}\right)$ is said to be an intermediate space between $D(A)$ and $\mathbb{X}$, if $D(A) \subset \mathbb{X}_{\alpha} \subset \mathbb{X}$ and there is a constant $C>0$ such that

$$
\|x\|_{\alpha} \leq C\|x\|^{1-\alpha}\|x\|_{A^{\prime}}^{\alpha} \quad x \in D(A)
$$

where $\|\cdot\|_{A}$ is the graph norm of $A$.

Concrete examples of $\mathbb{X}_{\alpha}$ include $D\left(A^{\alpha}\right)$ for $\alpha \in(0,1)$, the domains of the fractional powers of $A$, the real interpolation spaces $D_{A}(\alpha, \infty), \alpha \in(0,1)$, defined as follows:

$$
\begin{gathered}
D_{A}(\alpha, \infty):=\left\{x \in \mathbb{X}:[x]_{\alpha}=\sup _{0<t \leq 1}\left\|t^{1-\alpha}(A-\omega I) e^{-\omega t} T(t) x\right\|<\infty\right\}, \\
\|x\|_{\alpha}=\|x\|+[x]_{\alpha}
\end{gathered}
$$

and the abstract Hölder spaces $D_{A}(\alpha):=\overline{D(A)}^{\|\cdot\|_{\alpha}}$.

Lemma 2.5. For the hyperbolic analytic semigroup $(T(t))_{t \geq 0}$, there exist constants $C(\alpha)>0, \delta>$ $0, M(\alpha)>0$, and $\gamma>0$ such that

$$
\begin{gathered}
\|T(t) Q x\|_{\alpha} \leq C(\alpha) e^{\delta t}\|x\| \quad \text { for } t \leq 0, \\
\|T(t) P x\|_{\alpha} \leq M(\alpha) t^{-\alpha} e^{-\gamma t}\|x\| \quad \text { for } t>0 .
\end{gathered}
$$


Lemma 2.6 (see [9]). Let $0<\alpha<\beta<1$. For the hyperbolic analytic semigroup $(T(t))_{t \geq 0}$, there exist constants $c>0, \delta>0$, and $\gamma>0$ such that

$$
\begin{gathered}
\|A T(t) Q x\|_{\alpha} \leq c e^{\delta t}\|x\|_{\beta} \quad \text { for } t \leq 0, \\
\|A T(t) P x\|_{\alpha} \leq c t^{\beta-\alpha-1} e^{-\gamma t}\|x\|_{\beta} \quad \text { for } t>0 .
\end{gathered}
$$

\section{Pseudo almost automorphic functions}

In this section, we recall some recent results on almost automorphic functions and pseudo almost automorphic functions. Let $C(\mathbb{R}, \mathbb{X})$ denote the collection of continuous functions from $\mathbb{R}$ into $\mathbb{X}$. Let $B C(\mathbb{R}, \mathbb{X})$ denote the Banach space of all $\mathbb{X}$-valued bounded continuous functions equipped with the sup norm $\|u\|_{\infty}:=\sup _{t \in \mathbb{R}}\|u(t)\|$ for each $u \in B C(\mathbb{R}, \mathbb{X})$. Similarly, $C(\mathbb{R} \times$ $\mathbb{X}, \mathbb{X})$ denotes the collection of continuous functions from $\mathbb{R} \times \mathbb{X}$ into $\mathbb{X}, B C(\mathbb{R} \times \mathbb{X}, \mathbb{X})$ denotes the collection of all bounded continuous functions $f: \mathbb{R} \times \mathbb{X} \mapsto \mathbb{X}$.

Definition 3.1 (see [8]). A function $f \in C(\mathbb{R}, \mathbb{X})$ is said to be almost automorphic if for every sequence of real numbers $\left(\sigma_{n}\right)_{n \in \mathbb{N}}$, there exists a subsequence $\left(s_{n}\right)_{n \in \mathbb{N}}$ such that

$$
\lim _{m \rightarrow+\infty} \lim _{n \rightarrow+\infty} f\left(t+s_{n}-s_{m}\right)=f(t) \quad \text { for each } t \in \mathbb{R} .
$$

This limit means that

$$
g(t)=\lim _{n \rightarrow+\infty} f\left(t+s_{n}\right)
$$

is well defined for each $t \in \mathbb{R}$, and

$$
f(t)=\lim _{n \rightarrow+\infty} g\left(t-s_{n}\right)
$$

for each $t \in \mathbb{R}$. The collection of all such functions will be denoted by $\mathrm{AA}(\mathbb{R}, \mathbb{X})$.

Theorem 3.2 (see [7]). Assume $f, g: \mathbb{R} \mapsto \mathbb{X}$ are almost automorphic, and $\lambda$ is any scalar. Then the following holds true:

(1) $f+g, \lambda f, f_{\tau}(t):=f(t+\tau)$ and $\widehat{f}(t):=f(-t)$ are almost automorphic;

(2) the range $R_{f}$ of $f$ is precompact, so $f$ is bounded;

(3) if $\left\{f_{n}\right\}$ is a sequence of almost automorphic functions and $f_{n} \rightarrow f$ uniformly on $\mathbb{R}$, then $f$ is almost automorphic.

Theorem 3.3 (see [7]). If one equips $A A(\mathbb{R}, \mathbb{X})$ with the sup norm $\|u\|_{\infty}=\sup _{t \in \mathbb{R}}\|u(t)\|$, then $A A(\mathbb{R}, \mathbb{X})$ turns out to be a Banach space.

One sets

$$
\mathrm{AA}_{0}(\mathbb{R}, \mathbb{X}):=\left\{f \in B C(\mathbb{R}, \mathbb{X}): \lim _{r \rightarrow \infty} \frac{1}{2 r} \int_{-r}^{r}\|f(s)\| d s=0\right\}
$$


Definition 3.4 (see [2]). A function $f \in B C(\mathbb{R}, \mathbb{X})$ is said to be pseudo almost automorphic if it can be decomposed as $f=g+\phi$, where $g \in \mathrm{AA}(\mathbb{R}, \mathbb{X})$ and $\phi \in \mathrm{AA}_{0}(\mathbb{R}, \mathbb{X})$. The collection of such functions will be denoted by $\operatorname{PAA}(\mathbb{R}, \mathbb{X})$.

Theorem 3.5 (see [3]). If one equips $P A A(\mathbb{R}, \mathbb{X})$ with the sup norm $\|u\|_{\infty}=\sup _{t \in \mathbb{R}}\|u(t)\|$, then $P A A(\mathbb{R}, \mathbb{X})$ turns out to be a Banach space.

Definition 3.6 (see [2]). A function $f \in C(\mathbb{R} \times \mathbb{X}, \mathbb{X})$ is said to be almost automorphic if $f \in C(\mathbb{R} \times \mathbb{X}, \mathbb{X})$ is almost automorphic in $t \in \mathbb{R}$ uniformly for all $x \in K$, where $K$ is any bounded subset of $\mathbb{X}$. That is to say, for every sequence of real numbers $\left(\sigma_{n}\right)_{n \in \mathbb{N}}$ there exists a subsequence $\left(s_{n}\right)_{n \in \mathbb{N}}$ such that

$$
g(t, x)=\lim _{n \rightarrow+\infty} f\left(t+s_{n}, x\right)
$$

is well defined in $t \in \mathbb{R}$, for all $x \in K$, and

$$
f(t, x)=\lim _{n \rightarrow+\infty} g\left(t-s_{n}, x\right)
$$

for each $t \in \mathbb{R}$ and $x \in K$. Denote by $\mathrm{AA}(\mathbb{R} \times \mathbb{X}, \mathbb{X})$ the collection of all such functions. that

One also defines $\mathrm{AA}_{0}(\mathbb{R} \times \mathbb{X}, \mathbb{X})$ as the collection of functions $f \in B C(\mathbb{R} \times \mathbb{X}, \mathbb{X})$ such

$$
\lim _{r \rightarrow \infty} \frac{1}{2 r} \int_{-r}^{r}\|f(t, x)\| d t=0
$$

uniformly for any bounded subset of $\mathbb{X}$.

Definition 3.7 (see [2]). A function $f \in B C(\mathbb{R} \times \mathbb{X}, \mathbb{X})$ is said to be pseudo almost automorphic if it can be decomposed as $f=g+\phi$, where $g \in \mathrm{AA}(\mathbb{R} \times \mathbb{X}, \mathbb{X})$ and $\phi \in \mathrm{AA}_{0}(\mathbb{R} \times \mathbb{X}, \mathbb{X})$. The collection of such functions will be denoted by $\operatorname{PAA}(\mathbb{R} \times \mathbb{X}, \mathbb{X})$.

Theorem 3.8 (see [2]). Assume $f=g+\phi \in P A A(\mathbb{R} \times \mathbb{X}, \mathbb{X})$ with $g(t, x) \in A A(\mathbb{R} \times \mathbb{X}, \mathbb{X})$, $\phi(t, x) \in A A_{0}(\mathbb{R} \times \mathbb{X}, \mathbb{X})$, satisfying the following conditions:

(1) $g(t, x)$ is uniformly continuous in any bounded subset $K \subset \mathbb{X}$ uniformly for $t \in \mathbb{R}$;

(2) $f(t, x)$ is uniformly continuous in any bounded subset $K \subset \mathbb{X}$ uniformly for $t \in \mathbb{R}$.

If $x(\cdot) \in P A A(\mathbb{R}, \mathbb{X})$, then $f(\cdot, x(\cdot)) \in P A A(\mathbb{R}, \mathbb{X})$.

Remark 3.9. If $\mathbb{X}_{\alpha}$ for $\alpha \in(0,1)$ is an intermediate space between $D(A)$ and $\mathbb{X}, B C\left(\mathbb{R}, \mathbb{X}_{\alpha}\right)$, $\mathrm{AA}\left(\mathbb{R}, \mathbb{X}_{\alpha}\right), \operatorname{PAA}\left(\mathbb{R}, \mathbb{X}_{\alpha}\right)$ are equipped with the $\alpha$-sup norm: $\|u\|_{\infty, \alpha}=\sup _{t \in \mathbb{R}}\|u(t)\|_{\alpha}$, then they all constitute Banach spaces in view of Definition 2.4, Theorems 3.3 and 3.5.

Theorem 3.10 (see [6]). Let $u \in P A A(\mathbb{R}, \mathbb{Y}), B \in B(\mathbb{Y}, \mathbb{X})$. If $v(t):=B u(t)$ for each $t \in \mathbb{R}$, then $v \in P A A(\mathbb{R}, \mathbb{X})$. 


\section{Pseudo almost automorphic mild solutions}

In this section, combining Theorem 3.10 with composition theorem of pseudo almost automorphic functions (Theorem 3.8) and completeness of the space (PAA $\left.(\mathbb{R}, \mathbb{X}),\|\cdot\|_{\infty}\right)$ (Theorem 3.5), we will establish the existence and uniqueness theorem of pseudo almost automorphic mild solutions to (1.1) under the following assumptions.

(H1) For $0<\alpha<\beta<1, \mathbb{X}_{\beta} \hookrightarrow \mathbb{X}_{\alpha} \hookrightarrow \mathbb{X}$ are continuously embedded and there exist $k_{0}>0, k(\alpha)>0$ such that $\|u\| \leq k_{0}\|u\|_{\beta},\|u\|_{\alpha} \leq k(\alpha)\|u\|_{\beta}$ for each $u \in \mathbb{X}_{\beta}$.

$(\mathrm{H} 2) A$ is a sectorial linear operator on a Banach space $\mathbb{X}$ and $\sigma(A) \cap i \mathbb{R}=\varnothing$.

(H3) $f=\mathcal{M}+\varphi \in \operatorname{PAA}\left(\mathbb{R} \times \mathbb{X}, \mathbb{X}_{\beta}\right)$ with $\mathcal{M} \in \mathrm{AA}\left(\mathbb{R} \times \mathbb{X}, \mathbb{X}_{\beta}\right), \varphi \in \mathrm{AA}_{0}\left(\mathbb{R} \times \mathbb{X}, \mathbb{X}_{\beta}\right)$ and $g=\mathcal{N}+\psi \in \operatorname{PAA}(\mathbb{R} \times \mathbb{X}, \mathbb{X})$ with $\mathcal{N} \in \mathrm{AA}(\mathbb{R} \times \mathbb{X}, \mathbb{X}), \psi \in \mathrm{AA}_{0}(\mathbb{R} \times \mathbb{X}, \mathbb{X})$.

(H4) The functions $f, g$ are uniformly Lipschitz with respect to the second argument in the sense that: there exists $L>0$ such that

$$
\begin{gathered}
\|f(t, x)-f(t, y)\|_{\beta} \leq L\|x-y\|, \\
\|g(t, x)-g(t, y)\| \leq L\|x-y\|,
\end{gathered}
$$

for all $t \in \mathbb{R}$ and $x, y \in \mathbb{X}$.

(H5) $\mathcal{M}, \mathcal{N}$ are uniformly continuous in any bounded subset $K \subset \mathbb{X}$ uniformly for $t \in \mathbb{R}$.

(H6) The operators $B, C \in B\left(\mathbb{X}_{\alpha}, \mathbb{X}\right)$ and $\bar{\omega}=\max \left(\|B\|_{B\left(\mathbb{X}_{\alpha}, \mathbb{X}\right)},\|C\|_{B\left(\mathbb{X}_{\alpha}, \mathbb{X}\right)}\right)$.

Definition 4.1 (see [9]). A function $u \in B C\left(\mathbb{R}, \mathbb{X}_{\alpha}\right)$ is said to be a mild solution to (1.1) if $s \rightarrow A T(t-s) P f(s, B u(s))$ is integrable on $(-\infty, t), s \rightarrow A T(t-s) Q f(s, B u(s))$ is integrable on $(t,+\infty)$ and

$$
\begin{aligned}
u(t)= & -f(t, B u(t))-\int_{-\infty}^{t} A T(t-s) P f(s, B u(s)) d s+\int_{t}^{+\infty} A T(t-s) Q f(s, B u(s)) d s \\
& +\int_{-\infty}^{t} T(t-s) \operatorname{Pg}(s, C u(s)) d s-\int_{t}^{+\infty} T(t-s) Q g(s, C u(s)) d s
\end{aligned}
$$

for each $t \in \mathbb{R}$.

Lemma 4.2. Let assumptions (H1)-(H6) hold. Consider the nonlinear operator $\Lambda_{1}$ defined by

$$
\left(\Lambda_{1} u\right)(t)=\int_{-\infty}^{t} A T(t-s) P f(s, B u(s)) d s-\int_{t}^{+\infty} A T(t-s) Q f(s, B u(s)) d s
$$

Then, $\Lambda_{1}$ maps $P A A\left(\mathbb{R}, \mathbb{X}_{\alpha}\right)$ into itself. 
Proof. From (H1), (H3), (H4), (H5), we deduce that $f=\mathcal{M}+\varphi \in \operatorname{PAA}(\mathbb{R} \times \mathbb{X}, \mathbb{X})$ with $\mathcal{M} \in$ $\mathrm{AA}(\mathbb{R} \times \mathbb{X}, \mathbb{X}), \varphi \in \mathrm{AA}_{0}(\mathbb{R} \times \mathbb{X}, \mathbb{X})$, satisfying the following conditions.

(1) $\mathcal{M}(t, x)$ is uniformly continuous in any bounded subset $K \subset \mathbb{X}$ uniformly for $t \in \mathbb{R}$.

(2) $f(t, x)$ is uniformly Lipschitz with respect to the second argument in the sense that there exists $k_{0} L>0$ such that

$$
\|f(t, x)-f(t, y)\| \leq k_{0} L\|x-y\|
$$

for all $t \in \mathbb{R}$ and $x, y \in \mathbb{X}$.

Let $u \in \operatorname{PAA}\left(\mathbb{R}, \mathbb{X}_{\alpha}\right)$. Since $B \in B\left(\mathbb{X}_{\alpha}, \mathbb{X}\right)$, it follows from Theorem 3.10 that $B u(\cdot) \in$ $\operatorname{PAA}(\mathbb{R}, \mathbb{X})$. Setting $h(\cdot)=f(\cdot, B u(\cdot))$ and applying Theorem 3.8, we get that $h \in \operatorname{PAA}(\mathbb{R}, \mathbb{X})$. Using (H1) and Theorem 3.10, we obtain that $h \in \operatorname{PAA}\left(\mathbb{R}, \mathbb{X}_{\beta}\right)$. Now, write $h=\xi+\eta$ where $\xi \in \mathrm{AA}\left(\mathbb{R}, \mathbb{X}_{\beta}\right)$ and $\eta \in \mathrm{AA}_{0}\left(\mathbb{R}, \mathbb{X}_{\beta}\right)$, then

$$
\begin{aligned}
\left(\Lambda_{1} u\right)(t)= & \int_{-\infty}^{t} A T(t-s) P \xi(s) d s+\int_{-\infty}^{t} A T(t-s) P \eta(s) d s \\
& +\int_{+\infty}^{t} A T(t-s) Q \xi(s) d s+\int_{+\infty}^{t} A T(t-s) Q \eta(s) d s .
\end{aligned}
$$

Set

$$
\begin{aligned}
& \Xi(t)=\int_{-\infty}^{t} A T(t-s) P \xi(s) d s+\int_{+\infty}^{t} A T(t-s) Q \xi(s) d s \\
& \Pi(t)=\int_{-\infty}^{t} A T(t-s) P \eta(s) d s+\int_{+\infty}^{t} A T(t-s) Q \eta(s) d s
\end{aligned}
$$

Next, we show that $\Xi \in \mathrm{AA}\left(\mathbb{R}, \mathbb{X}_{\alpha}\right)$ and $\Pi \in \mathrm{AA}_{0}\left(\mathbb{R}, \mathbb{X}_{\alpha}\right)$.

Now, to prove that $\Xi \in \mathrm{AA}\left(\mathbb{R}, \mathbb{X}_{\alpha}\right)$. Let us take a sequence $\left(\sigma_{n}\right)_{n \in \mathbb{N}}$ and show that there exists a subsequence $\left(s_{n}\right)_{n \in \mathbb{N}}$ such that

$$
\lim _{m \rightarrow+\infty} \lim _{n \rightarrow+\infty}\left\|\Xi\left(t+s_{n}-s_{m}\right)-\Xi(t)\right\|_{\alpha}=0 \quad \text { for each } t \in \mathbb{R}
$$

Since $\xi \in \mathrm{AA}\left(\mathbb{R}, \mathbb{X}_{\beta}\right)$, there exists a subsequence $\left(s_{n}\right)_{n \in \mathbb{N}}$ such that

$$
\lim _{m \rightarrow+\infty} \lim _{n \rightarrow+\infty}\left\|\xi\left(t+s_{n}-s_{m}\right)-\xi(t)\right\|_{\beta}=0 \quad \text { for each } t \in \mathbb{R}
$$


On the other hand, we have

$$
\begin{aligned}
\Xi\left(t+s_{n}-s_{m}\right)-\Xi(t)= & \int_{-\infty}^{\left(t+s_{n}-s_{m}\right)} A T\left(t+s_{n}-s_{m}-s\right) P \xi(s) d s-\int_{-\infty}^{t} A T(t-s) P \xi(s) d s \\
& +\int_{+\infty}^{t+s_{n}-s_{m}} A T\left(t+s_{n}-s_{m}-s\right) Q \xi(s) d s-\int_{+\infty}^{t} A T(t-s) Q \xi(s) d s \\
= & \int_{0}^{+\infty} A T(s) P \xi\left(t+s_{n}-s_{m}-s\right) d s-\int_{0}^{+\infty} A T(s) P \xi(t-s) d s \\
& -\left(\int_{-\infty}^{0} A T(s) Q \xi\left(t+s_{n}-s_{m}-s\right) d s-\int_{-\infty}^{0} A T(s) Q \xi(t-s) d s\right) \\
= & \int_{0}^{+\infty} A T(s) P\left[\xi\left(t+s_{n}-s_{m}-s\right)-\xi(t-s)\right] d s \\
& -\int_{-\infty}^{0} A T(s) Q\left[\xi\left(t+s_{n}-s_{m}-s\right)-\xi(t-s)\right] d s .
\end{aligned}
$$

Passing to the norm $\|\cdot\|_{\alpha}, 0<\alpha<1$, remembering the triangle inequalities, (2.7), then we obtain that

$$
\begin{aligned}
\left\|\Xi\left(t+s_{n}-s_{m}\right)-\Xi(t)\right\|_{\alpha} \leq & \int_{0}^{+\infty}\left\|A T(s) P\left[\xi\left(t+s_{n}-s_{m}-s\right)-\xi(t-s)\right]\right\|_{\alpha} d s \\
& +\int_{-\infty}^{0}\left\|A T(s) Q\left[\xi\left(t+s_{n}-s_{m}-s\right)-\xi(t-s)\right]\right\|_{\alpha} d s \\
\leq & c \int_{0}^{+\infty} s^{\beta-\alpha-1} e^{-\gamma s}\left\|\xi\left(t+s_{n}-s_{m}-s\right)-\xi(t-s)\right\|_{\beta} d s \\
& +c \int_{-\infty}^{0} e^{\delta s}\left\|\xi\left(t+s_{n}-s_{m}-s\right)-\xi(t-s)\right\|_{\beta} d s .
\end{aligned}
$$

Thus, (4.8) and Lebesgue dominated convergence theorem lead to (4.7), therefore, to $\Xi \in$ $\mathrm{AA}\left(\mathbb{R}, \mathbb{X}_{\alpha}\right)$. To finish the proof, we will prove that $\Pi \in \mathrm{AA}_{0}\left(\mathbb{R}, \mathbb{X}_{\alpha}\right)$. It is obvious that $\Pi \in$ $B C\left(\mathbb{R}, \mathbb{X}_{\alpha}\right)$, the left task is to show that

$$
\lim _{r \rightarrow \infty} \frac{1}{2 r} \int_{-r}^{r}\|\Pi(t)\|_{\alpha} d t=0
$$

Using (2.7), we have

$$
0 \leq \lim _{r \rightarrow \infty} \frac{1}{2 r} \int_{-r}^{r}\|\Pi(t)\|_{\alpha} d t \leq I+J
$$


where

$$
\begin{aligned}
I & :=\lim _{r \rightarrow \infty} \frac{c}{2 r} \int_{-r}^{r} d t \int_{-\infty}^{t}(t-s)^{\beta-\alpha-1} e^{-\gamma(t-s)}\|\eta(s)\|_{\beta} d s \\
& =\lim _{r \rightarrow \infty} \frac{c}{2 r} \int_{-r}^{r} d t \int_{0}^{+\infty} \sigma^{\beta-\alpha-1} e^{-\gamma \sigma}\|\eta(t-\sigma)\|_{\beta} d \sigma \\
& =\lim _{r \rightarrow \infty} c \int_{0}^{+\infty} \sigma^{\beta-\alpha-1} e^{-\gamma \sigma}\left[\frac{1}{2 r} \int_{-r}^{r}\|\eta(t-\sigma)\|_{\beta} d t\right] d \sigma, \\
J & :=\lim _{r \rightarrow \infty} \frac{c}{2 r} \int_{-r}^{r} d t \int_{t}^{+\infty} e^{\delta(t-s)}\|\eta(s)\|_{\beta} d s \\
& =\lim _{r \rightarrow \infty} \frac{c}{2 r} \int_{-r}^{r} d t \int_{-\infty}^{0} e^{\delta \sigma}\|\eta(t-\sigma)\|_{\beta} d \sigma \\
& =\lim _{r \rightarrow \infty} c \int_{-\infty}^{0} e^{\delta \sigma}\left[\frac{1}{2 r} \int_{-r}^{r}\|\eta(t-\sigma)\|_{\beta} d t\right] d \sigma .
\end{aligned}
$$

Then, by the Lebesgue dominated convergence theorem and the fact that $\eta \in \mathrm{AA}_{0}\left(\mathbb{R}_{,} \mathbb{X}_{\beta}\right)$, one has $I=J=0$. Hence, $\Pi \in \mathrm{AA}_{0}\left(\mathbb{R}, \mathbb{X}_{\alpha}\right)$ and we end the proof.

Lemma 4.3. Let assumptions (H2)-(H6) hold. Define the nonlinear operator $\Lambda_{2}$ by

$$
\left(\Lambda_{2} u\right)(t)=\int_{-\infty}^{t} T(t-s) \operatorname{Pg}(s, C u(s)) d s-\int_{t}^{+\infty} T(t-s) Q g(s, C u(s)) d s
$$

Then, $\Lambda_{2}$ maps PAA $\left(\mathbb{R}, \mathbb{X}_{\alpha}\right)$ into itself.

Proof. The proof is similar to that of Lemma 4.2, so we omit it.

Theorem 4.4. Under the assumptions (H1)-(H6), partial hyperbolic evolution equation (1.1) admits a unique pseudo almost automorphic mild solution if

$$
\Theta=L \bar{\omega}\left[k(\alpha)+\frac{c}{\delta}+\frac{C(\alpha)}{\delta}+\frac{c \Gamma(\beta-\alpha)}{\gamma^{\beta-\alpha}}+\frac{M(\alpha) \Gamma(1-\alpha)}{\gamma^{1-\alpha}}\right]<1 .
$$

Proof. Firstly, define the nonlinear operator $\Lambda$ on $B C\left(\mathbb{R}, \mathbb{X}_{\alpha}\right)$ by

$$
\begin{aligned}
(\Lambda u)(t)= & -f(t, B u(t))-\int_{-\infty}^{t} A T(t-s) P f(s, B u(s)) d s+\int_{t}^{+\infty} A T(t-s) Q f(s, B u(s)) d s \\
& +\int_{-\infty}^{t} T(t-s) \operatorname{Pg}(s, C u(s)) d s-\int_{t}^{+\infty} T(t-s) Q g(s, C u(s)) d s \\
= & -f(t, B u(t))-\left(\Lambda_{1} u\right)(t)+\left(\Lambda_{2} u\right)(t) .
\end{aligned}
$$


Let $u \in \operatorname{PAA}\left(\mathbb{R}, \mathbb{X}_{\alpha}\right)$, then $f(\cdot, B u(\cdot)) \in \operatorname{PAA}\left(\mathbb{R}, \mathbb{X}_{\beta}\right) \subset \operatorname{PAA}\left(\mathbb{R}, \mathbb{X}_{\alpha}\right)$, as proved in Lemma 4.2. Together with Lemmas 4.2 and 4.3 , it follows that the operator $\Lambda$ maps PAA $\left(\mathbb{R}, \mathbb{X}_{\alpha}\right)$ into itself. Secondly, we will show that $\Lambda$ admits a unique fixed point in $\operatorname{PAA}\left(\mathbb{R}, \mathbb{X}_{\alpha}\right)$.

Let $v, w \in \operatorname{PAA}\left(\mathbb{R}, \mathbb{X}_{\alpha}\right)$, then the triangle inequality reads

$$
\begin{aligned}
\|(\Lambda v)(t)-(\Lambda w)(t)\|_{\alpha} \leq & \|f(t, B v(t))-f(t, B w(t))\|_{\alpha}+\left\|\left(\Lambda_{1} v\right)(t)-\left(\Lambda_{1} w\right)(t)\right\|_{\alpha} \\
& +\left\|\left(\Lambda_{2} v\right)(t)-\left(\Lambda_{2} w\right)(t)\right\|_{\alpha}
\end{aligned}
$$

By (H1), (H5), and (H6), we obtain

$$
\|f(t, B v(t))-f(t, B w(t))\|_{\alpha} \leq k(\alpha) L\|B v(t)-B w(t)\| \leq k(\alpha) L \bar{\omega}\|v-w\|_{\infty, \alpha}
$$

By (H2), Lemma 2.6, (H5), and (H6), we obtain

$$
\begin{aligned}
\left\|\left(\Lambda_{1} v\right)(t)-\left(\Lambda_{1} w\right)(t)\right\|_{\alpha} \leq & \int_{-\infty}^{t}\|A T(t-s) P[f(s, B v(s))-f(s, B w(s))]\|_{\alpha} d s \\
& +\int_{t}^{+\infty}\|A T(t-s) Q[f(s, B v(s))-f(s, B w(s))]\|_{\alpha} d s \\
\leq & c L \bar{\omega}\|v-w\|_{\infty, \alpha} \int_{-\infty}^{t}(t-s)^{\beta-\alpha-1} e^{-\gamma(t-s)} d s \\
& +c L \bar{\omega}\|v-w\|_{\infty, \alpha} \int_{t}^{+\infty} e^{\delta(t-s)} d s \\
\leq & c L \bar{\omega}\|v-w\|_{\infty, \alpha}\left[\frac{\Gamma(\beta-\alpha)}{\gamma^{\beta-\alpha}}+\frac{1}{\delta}\right]
\end{aligned}
$$

Similarly, by (H2), Lemma 2.5, (H5), and (H6), we obtain

$$
\begin{aligned}
\left\|\left(\Lambda_{2} v\right)(t)-\left(\Lambda_{2} w\right)(t)\right\|_{\alpha} \leq & \int_{-\infty}^{t}\|T(t-s) P[g(s, C v(s))-g(s, C w(s))]\|_{\alpha} d s \\
& +\int_{t}^{+\infty}\|T(t-s) Q[g(s, C v(s))-g(s, C w(s))]\|_{\alpha} d s \\
\leq & M(\alpha) L \bar{\omega}\|v-w\|_{\infty, \alpha} \int_{-\infty}^{t}(t-s)^{-\alpha} e^{-\gamma(t-s)} d s \\
& +C(\alpha) L \bar{\omega}\|v-w\|_{\infty, \alpha} \int_{t}^{+\infty} e^{\delta(t-s)} d s \\
\leq & L \bar{\omega}\|v-w\|_{\infty, \alpha}\left[\frac{M(\alpha) \Gamma(1-\alpha)}{r^{1-\alpha}}+\frac{C(\alpha)}{\delta}\right] .
\end{aligned}
$$


Combining the above inequality together, we obtain $\|\Lambda v-\Lambda w\|_{\infty, \alpha} \leq \Theta\|v-w\|_{\infty, \alpha}$, where

$$
\Theta=L \bar{\omega}\left[k(\alpha)+\frac{c}{\delta}+\frac{C(\alpha)}{\delta}+\frac{c \Gamma(\beta-\alpha)}{\gamma^{\beta-\alpha}}+\frac{M(\alpha) \Gamma(1-\alpha)}{\gamma^{1-\alpha}}\right]
$$

Clearly, if $\Theta<1$, then the operator $\Lambda$ becomes a strict contraction on $\operatorname{PAA}\left(\mathbb{R}, \mathbb{X}_{\alpha}\right)$. Remembering that $\operatorname{PAA}\left(\mathbb{R}, \mathbb{X}_{\alpha}\right)$ equipped with the $\alpha$-sup norm: $\|u\|_{\infty, \alpha}=\sup _{t \in \mathbb{R}}\|u(t)\|_{\alpha}$ is a Banach space by Remark 3.9, the classical Banach fixed-point theorem leads to the desired conclusion.

\section{Application}

Example 5.1. Take $\mathbb{X}:=C[0, \pi]$ equipped with the sup norm. Define the operator $A$ by

$$
A \varphi(\xi):=\varphi^{\prime \prime}(\xi)+a \varphi(\xi), \quad \forall \xi \in(0, \pi), \varphi \in D(A)
$$

where $D(A):=\left\{\varphi \in C^{2}[0, \pi], \varphi(0)=\varphi(\pi)=0\right\} \subset C[0, \pi]$ and $a \in \mathbb{R}$ is a constant.

Clearly, $A$ is sectorial, and hence is the generator of an analytic semigroup. If $a \neq n^{2}$, then $A$ generates a hyperbolic analytic semigroup $(T(t))_{t \geq 0}$ on $\mathbb{X}$.

Let

$$
\mathbb{X}_{\alpha}=D_{A}(\alpha, \infty)= \begin{cases}C^{2 \alpha}[0, \pi], & \text { if } 0<\alpha<\frac{1}{2} \\ C_{v}^{2 \alpha}[0, \pi], & \text { if } \frac{1}{2}<\alpha<1,\end{cases}
$$

where $C_{v}^{2 \alpha}[0, \pi]=\left\{\varphi \in C^{2 \alpha}[0, \pi], \varphi(0)=\varphi(\pi)=0\right\}$ (see [10] for more details).

Define the operator $C$ by

$$
C \varphi(\xi):=q(\xi) \varphi(\xi), \quad \forall \xi \in(0, \pi), \varphi \in D(C),
$$

where $D(C):=\{\varphi \in C[0, \pi], \varphi(0)=\varphi(\pi)=0\} \subset C[0, \pi]$, then $\mathbb{X}_{\alpha} \subset D(C)$ and $\|C\|_{B\left(\mathbb{X}_{\alpha}, \mathbb{X}\right)}=$ $\|q\|_{\infty}=\sup _{\xi \in[0, \pi]} q(\xi)$.

Let

$$
g(t, u)=u \sin \frac{1}{2+\cos t+\cos \sqrt{2} t}+\max _{k \in \mathbb{Z}}\left\{e^{-\left(t \pm k^{2}\right)^{2}}\right\} \cos u, \quad t \in \mathbb{R}, u \in \mathbb{X},
$$

then $g \in \operatorname{PAA}(\mathbb{R} \times \mathbb{X}, \mathbb{X}), g$ is Lipschitz with respect to the second argument with $L=$ 2, $\mathcal{N}(t, u)=u \sin (1 /(2+\cos t+\cos \sqrt{2} t))$ is uniformly continuous in any bounded subset $K \subset \mathbb{X}$ uniformly for $t \in \mathbb{R}$. 
Now the above discussion can be formulated as follows.

Proposition 5.2. Let $g(t, u)=u \sin (1 /(2+\cos t+\cos \sqrt{2} t))+\max _{k \in \mathbb{Z}}\left\{e^{-\left(t \pm k^{2}\right)^{2}}\right\} \cos u, t \in \mathbb{R}, u \in$ $\mathbb{X}$. Suppose that the constant $a \neq n^{2}$ for $n \in \mathbb{N}$. Then, nonlinear heat equation (1.2) admits a unique $\mathbb{X}_{\alpha}$-valued pseudo almost automorphic mild solution if

$$
2\|q\|_{\infty}\left[\frac{C(\alpha)}{\delta}+\frac{M(\alpha) \Gamma(1-\alpha)}{\gamma^{1-\alpha}}\right]<1
$$

\section{Appendix}

Proof of Theorem 3.10. Write $u=\phi+\psi$ where $\phi \in \mathrm{AA}(\mathbb{R}, \mathbb{Y})$ and $\psi \in \mathrm{AA}_{0}(\mathbb{R}, \mathbb{Y})$, then

$$
v(t)=B \phi(t)+B \psi(t)
$$

Set

$$
\Phi(t)=B \phi(t), \quad \Psi(t)=B \psi(t) .
$$

We are going to prove that $\Phi \in \mathrm{AA}(\mathbb{R}, \mathbb{X})$ and $\Psi \in \mathrm{AA}_{0}(\mathbb{R}, \mathbb{X})$. that

Let us take a sequence $\left(\sigma_{n}\right)_{n \in \mathbb{N}}$ and show that there exists a subsequence $\left(s_{n}\right)_{n \in \mathbb{N}}$ such

$$
\lim _{m \rightarrow+\infty} \lim _{n \rightarrow+\infty}\left\|\Phi\left(t+s_{n}-s_{m}\right)-\Phi(t)\right\|=0 \quad \text { for each } t \in \mathbb{R} .
$$

Since $\phi \in \mathrm{AA}(\mathbb{R}, \mathbb{Y})$, there exists a subsequence $\left(s_{n}\right)_{n \in \mathbb{N}}$ such that

$$
\lim _{m \rightarrow+\infty} \lim _{n \rightarrow+\infty}\left\|\phi\left(t+s_{n}-s_{m}\right)-\phi(t)\right\|_{\mathbb{Y}}=0 \quad \text { for each } t \in \mathbb{R} .
$$

On the other hand, we have

$$
0 \leq\left\|\Phi\left(t+s_{n}-s_{m}\right)-\Phi(t)\right\|=\left\|B \phi\left(t+s_{n}-s_{m}\right)-B \phi(t)\right\| \leq\|B\|_{B(\mathbb{Y}, \mathbb{X})}\left\|\phi\left(t+s_{n}-s_{m}\right)-\phi(t)\right\|_{\mathbb{Y}} .
$$

Thus, (A.4) leads to (A.3), therefore, to $\Phi \in \mathrm{AA}(\mathbb{R}, \mathbb{X})$.

To complete the proof, we will prove that $\Psi \in \mathrm{AA}_{0}(\mathbb{R}, \mathbb{X})$. In fact, it is easy to check that $\Psi \in B C(\mathbb{R}, \mathbb{X})$ and

$$
\lim _{r \rightarrow \infty} \frac{1}{2 r} \int_{-r}^{r}\|\Psi(t)\| d t \leq\|B\|_{B(\mathbb{Y}, \mathbb{X})} \lim _{r \rightarrow \infty} \frac{1}{2 r} \int_{-r}^{r}\|\psi(t)\|_{\mathbb{Y}} d t=0
$$

Hence, $\Psi \in \mathrm{AA}_{0}(\mathbb{R}, \mathbb{X})$ and we end the proof. 


\section{Acknowledgments}

The authors would like to thank referees for their helpful suggestions. This work is supported by the National Science Foundation of China (60771026), the Special Scientific Research Foundation for the Subjects of Doctors in University (20060110005), and the Program for New Century Excellent Talents in University (NCET050271).

\section{References}

[1] T. Diagana, "Existence of $p$-almost automorphic mild solution to some abstract differential equations," International Journal of Evolution Equations, vol. 1, no. 1, pp. 57-67, 2005.

[2] J. Liang, J. Zhang, and T.-J. Xiao, "Composition of pseudo almost automorphic and asymptotically almost automorphic functions," Journal of Mathematical Analysis and Applications, vol. 340, no. 2, pp. 1493-1499, 2008.

[3] T.-J. Xiao, J. Liang, and J. Zhang, "Pseudo almost automorphic solutions to semilinear differential equations in Banach spaces," Semigroup Forum, vol. 76, no. 3, pp. 518-524, 2008.

[4] T. Diagana, G. M. N'Guérékata, and N. Van Minh, "Almost automorphic solutions of evolution equations," Proceedings of the American Mathematical Society, vol. 132, no. 11, pp. 3289-3298, 2004.

[5] R. A. Johnson, "A linear, almost periodic equation with an almost automorphic solution," Proceedings of the American Mathematical Society, vol. 82, no. 2, pp. 199-205, 1981.

[6] Z. R. Hu and Z. Jin, "Pseudo almost automorphic mild solutions to some classes of partial evolution equations," submitted to Nonlinear Analysis: Real World Applications.

[7] G. M. N'Guérékata, Almost Automorphic and Almost Periodic Functions in Abstract Spaces, Kluwer Academic/Plenum, New York, NY, USA, 2001.

[8] G. M. N’Guérékata, Topics in Almost Automorphy, Springer, New York, NY, USA, 2005.

[9] T. Diagana, "Existence of pseudo almost periodic solutions to some classes of partial hyperbolic evolution equations," Electronic Journal of Qualitative Theory of Differential Equations, no. 3, pp. 1-12, 2007.

[10] A. Lunardi, Analytic Semigroups and Optimal Regularity in Parabolic Problems, vol. 16 of Progress in Nonlinear Differential Equations and Their Applications, Birkhäuser, Basel, Switzerland, 1995.

[11] K.-J. Engel and R. Nagel, One-Parameter Semigroups for Linear Evolution Equations, vol. 194 of Graduate Texts in Mathematics, Springer, New York, NY, USA, 2000. 


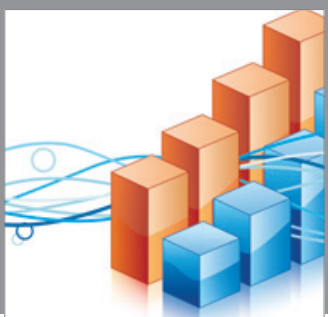

Advances in

Operations Research

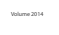

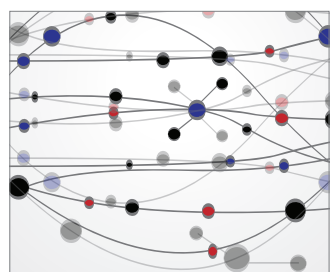

\section{The Scientific} World Journal
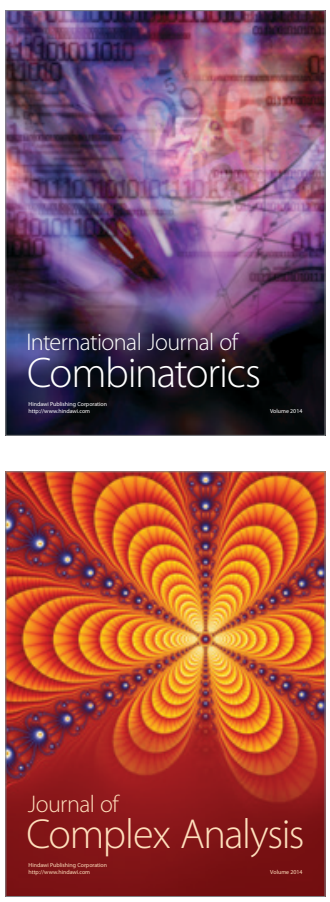

International Journal of

Mathematics and

Mathematical

Sciences
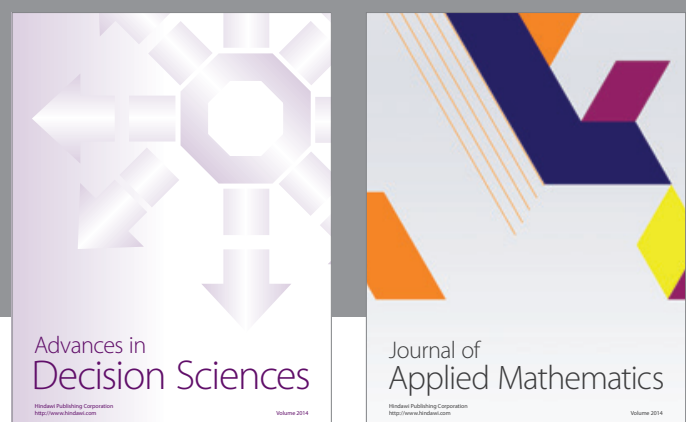

Journal of

Applied Mathematics
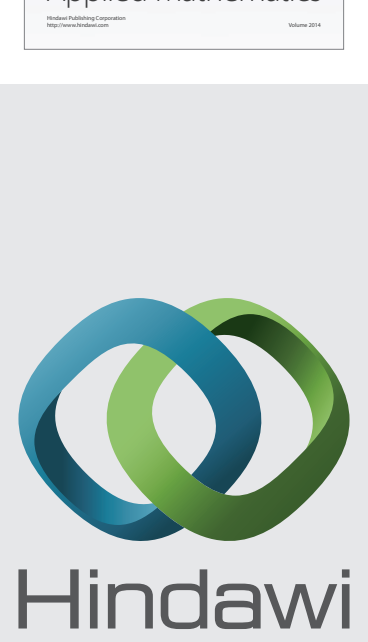

Submit your manuscripts at http://www.hindawi.com
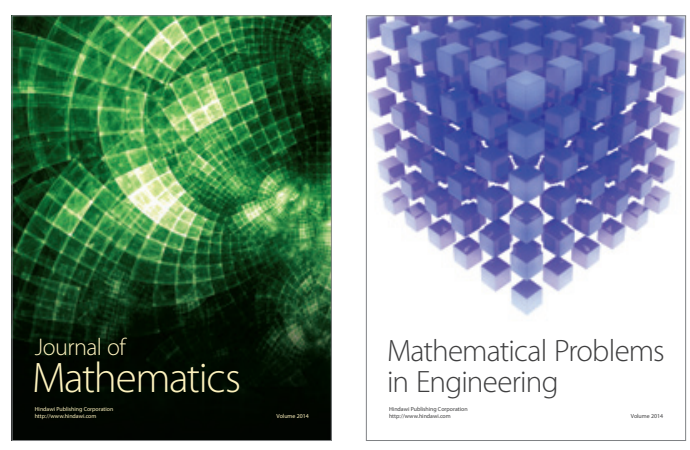

Mathematical Problems in Engineering
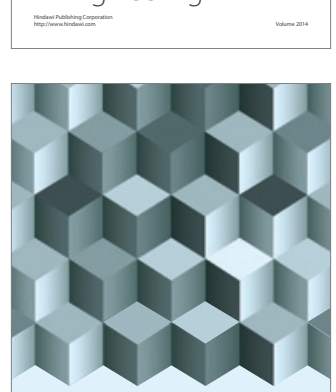

Journal of

Function Spaces
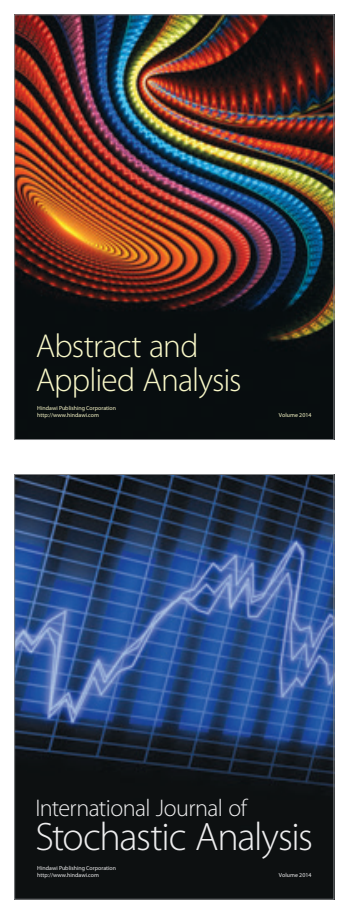

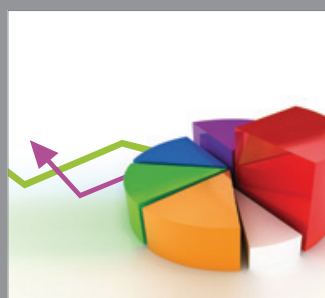

ournal of

Probability and Statistics

Promensencen
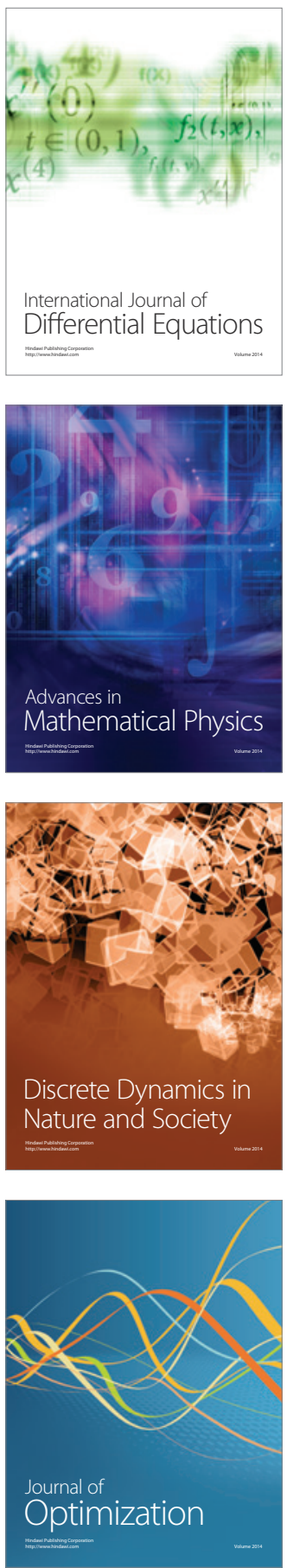